\title{
Ozone Production with High Voltage in a Mixed Core Mesh Coil
}

\section{Producción De Ozono Con Alto Voltaje En Un Núcleo Mixto Bobinamalla}

\author{
Chávez Velasco Iván Fabricio \\ ESPOCH, Ciencias, Escuela de Física y Matemáticas, Riobamba, Ecuador
}

I International Congress of Science and Technology Morona Santiago-CICTMS 2020

Corresponding Author:

Chávez Velasco Iván Fabricio ivan.chavez0697@gmail.com

Published: 29 August 2021

Production and Hosting by Knowledge $E$

(c) Chávez Velasco Iván Fabricio. This article is distributed under the terms of the Creative Commons Attribution License, which permits unrestricted use and redistribution provided that the original author and source are credited.

\section{Abstract}

The ozone is a gas composed by three molecules of oxygen which has a great oxidative capacity. This gas can be generated by a nucleus with two electrodes through high voltage in a phenomenon known as corona effect. Actually the conventional nucleus of ozone generators use plane electrodes with a dielectric in the middle of both. A nucleus composed by a mixed ionizador (coil and mesh) is presented as an alternative for an ozone machine in order to take advantage of the individual benefits of each electrode due to its shape and efficiency. A solenoid acts better without dielectric, reducing the consumption, and a mesh improves the air flux. As a result, there is a good production of ozone using this combination of electrodes. However, there are no studies that demonstrate that it is possible to use different types of electrodes in the same ionizador, for this reason in this study will be detected the production of ozone by corona effect in the proposed nucleus and will be approximated its concentration in a closed volume.

Keywords: Ozone, corona effect, high voltage, generator, ionization

\section{Resumen}

El ozono es un gas compuesto por tres moléculas de oxígeno que tiene una gran capacidad oxidativa. Este gas puede ser generado en un núcleo con dos electrodos mediante el uso de altos voltajes en un fenómeno conocido como efecto corona. Actualmente los núcleos de los generadores de ozono convencionales utilizan electrodos planos con un dieléctrico en medio de ellos. Un núcleo compuesto por un ionizador mixto (bobina y malla) se presenta como una alternativa para un ozonificador, con el fin de aprovechar las ventajas individuales de cada electrodo debido a su forma y eficiencia. Un solenoide actúa mejor sin dieléctrico, disminuyendo el consumo, y una malla mejora el flujo de aire. Lo que resulta en una buena producción de ozono utilizando esta combinación de electrodos. Sin embargo, no existen estudios anteriores que demuestran que se puede utilizar diferentes tipos de electrodos en un mismo ionizador, por esta razón en el presente estudio se detectará la producción de ozono por efecto corona en el núcleo mixto propuesto y aproximamos su concentración en un volumen cerrado.

Palabras Clave: Ozono, efecto corona, alto voltaje, generador, ionización 


\section{Introducción}

El Ozono es un gas que se puede encontrar en la naturaleza en su estado natural. Posee la forma triatómica del oxígeno por lo que su fórmula puede expresarse como $O \_3$, caracterizada por ser una molécula polar e incolora, capaz de oxidar compuestos orgánicos [1, 2]. El ozono al poseer una gran actividad oxidativa puede actuar sobre bacterias más rápido que el cloro [3] e incluso ha demostrado ser útil en el tratamiento de aguas debido a su efecto antimicrobiano [4]. Este gas se forma cuando un átomo de oxígeno se une a una molécula de oxígeno $\mathrm{O}_{2}$, sin embargo esta reacción no ocurre de manera espontánea [5]. Para producir una molécula de ozono primero se debe bombardear con energía a las moléculas de oxígeno, esto rompe su enlace covalente formando iones de oxígeno, que luego se agrupan con moléculas de oxígeno para formar moléculas de ozono [6]. [Energ $a+O \unrhd \_2=>2 O^{\wedge}-+$ Energ $a$

$$
O^{\wedge}-+O \_2=>O \_3 \text {. }
$$

En el caso del ozono atmosférico esta energía que rompe el enlace proviene de la radiación ultravioleta sobre la troposfera [8]. De igual manera, se puede conseguir esta energía extra a través de una descarga por efecto corona. Mediante este método, se puede generar ozono a partir de un flujo de aire o oxígeno entre dos electrodos [8].

El efecto corona ocurre cuando en dos conductores existe una acumulación de cargas que saturan el medio circundante, entonces el aire de los alrededores se vuelve ligeramente conductor [9]. El alto voltaje entre los electrodos acelera a los electrones, lo que incrementa su energía cinética y causa choques con la fuerza suficiente para romper la molécula de oxígeno [10].

El voltaje mínimo en el que ocurre el efecto corona es conocido como voltaje crítico disruptivo $\left(V \_d o\right.$ ) el cual depende de factores ambientales y estructurales de los conductores. En general puede expresarse mediante la siguiente expresión matemática:

$$
V_{d o}=g_{o} \delta m_{o} r \ln \frac{d}{r},
$$

$g_{o}$ es la resistencia dieléctrica del aire, $\delta m \_o$ son términos que dependen del ambiente [11]. De esta ecuación podemos despejar la distancia entre los conductores.

$$
d=r e^{\wedge}\left(V \_d o / k\right),
$$

en esta segunda ecuación $\mathrm{k}=g \_o \delta m \_o r$ y el $\mathrm{r}$ representa el radio de los conductores.

Por lo general, los generadores de ozono basados en efecto corona suelen usar electrodos planos con un dieléctrico en medio de ellos [3-6, 9-12] Además de este diseño también han aparecido nuevos estudios donde la estructura del núcleo cambia demostrando una mejor eficiencia. Puede verse que bajo ciertos niveles de voltaje la producción de ozono es mejor sin un dieléctrico entre electrodos sinusoidales [14]. En otro estudio se revelaron mejoras en la eficiencia si se trabaja con electrodos tipo malla con la ventaja de tener un mejor flujo de aire [15].

El objetivo del estudio presentado es demostrar que se puede crear un núcleo formado por electrodos de diferente estructura para generar ozono. El sistema propuesto posee las ventajas de no necesitar un dieléctrico, por lo que el consumo de voltaje es 
menor y además tiene un mayor flujo de aire a través del núcleo debido a que uno de los electrodos es de tipo malla. Esta es solo una de las posibles combinaciones que se podrían hacer para crear un núcleo ionizador de ozono.

\section{Materiales y Métodos}

La Figura 1 muestra un diagrama esquemático de la configuración del generador de ozono. La fuente de alimentación para el ozonificador será de corriente alterna de 120, el cual se conecta a un transformador reductor de voltaje. Luego la corriente pasa a través de un rectificador de corriente con salida de corriente continua. Dicha corriente pasa a través de un segundo transformador tipo flyback el cual elevará el voltaje hasta 14000v. El alto voltaje está conectado al núcleo donde se crea una alta diferencia de potencial necesaria para producir el efecto corona.

El transformador flyback se obtuvo de un televisor antiguo de 15 pulgadas y que funcionaba a blanco y negro. El flyback estaba en perfecto estado y se usó el mismo circuito del televisor para realizar las conexiones. Por lo que el consumo energético de este elevador puede ser aproximado al mismo consumo de dicho televisor el cual es de $55 \mathrm{~W}$.

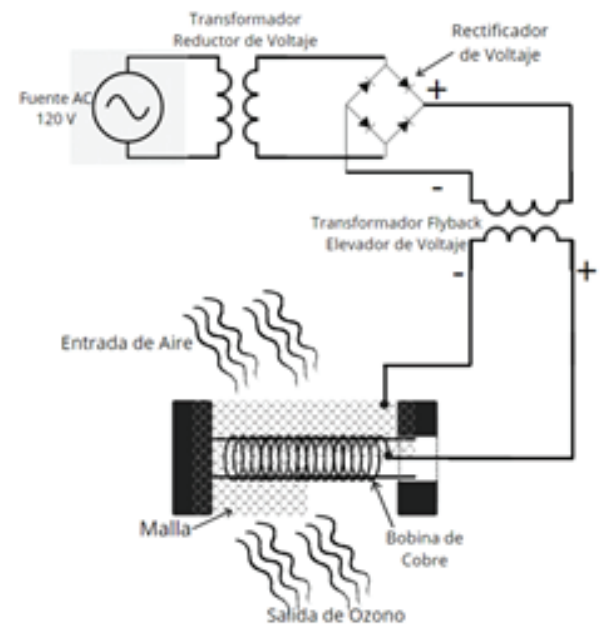

Figure 1

Configuración experimental del generador de ozono.

Utilizamos la ecuación 2 para determinar el espacio que debe haber entre los electrodos dentro del núcleo considerando que $\mathrm{k}=2555$ y el radio se reemplaza con el correspondiente al calibre del cable que está hecho el solenoide. De esta manera la distancia necesaria entre los electrodos para que se produzca el efecto corona es de $3.11 \mathrm{~cm}$. Con esta consideración construimos el ionizador.

El núcleo está conformado por un electrodo central sinusoidal de 28 espiras, hecho con cobre de calibre 10, envuelto sobre tubo de PVC de $13.5 \mathrm{~cm}$ de largo y $3 \mathrm{~cm}$ diámetro. El electrodo exterior está diseñado con una malla de aluminio en forma de cilindro alrededor del eje central, tiene el mismo largo del tubo de PVC y su diámetro 
es de $8 \mathrm{~cm}$. Cada uno de los electrodos está conectado a uno de los polos de salida del flyback elevador de voltaje.

En el experimento propuesto se ha conectado el polo positivo a la bobina interna mientras que el polo negativo está conectado a la malla, de esta manera dentro del cilindro coaxial se genera una diferencia de potencial capaz de generar ozono a partir de la entrada de aire de los alrededores.

Para realizar la detección de ozono se utilizará el método por difusión, el cual se basa en la reacción que tiene el ozono con una mezcla de yoduro de potasio y agua. La estequiometría de la reacción nos dice que un mol de $O \_3$ puede liberar un mol de Yodo [16].

$$
O \_3+3 K I+H \_2 O=>I \_2+O \_2+2 K O H \text {. }
$$

El sistema de medición consiste en utilizar tiras de papel con esta mezcla sensible al ozono, la cual cambia de coloración con su presencia, tornándose en un color amarillomarrón [17].

\section{Table 1}

Evaluación de lectura por difusión de ozono en interiores [17].

\begin{tabular}{|c|c|c|}
\hline Código & Color & Ozono $\left(\mu \mathrm{g} / \mathrm{m}^{3}\right)$ \\
\hline A & & $30-90$ \\
B & $\bigcirc$ & $90-150$ \\
C & $\bigcirc$ & $150-210$ \\
D & $\bigcirc$ & 210 o más \\
\hline
\end{tabular}

Para realizar la detección de ozono en el presente estudio hemos aislado el núcleo en el interior de un contenedor de tres litros, dentro del mismo se coloca una tira de papel impregnada con la solución de yoduro de potasio. Luego de diferentes intervalos de tiempo se retira el papel y se compara el color con la Tabla 1, de esta manera podemos tener una aproximación de la concentración de ozono en el interior del envase. En un contenedor diferente colocamos una tira con la mezcla pero en el interior no tendrá el generador de ozono durante 25 min; con el fin de tener una muestra testigo. La manera en que se construyó el experimento para detectar ozono se puede apreciar en la siguiente figura.

a)

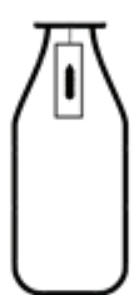

b)

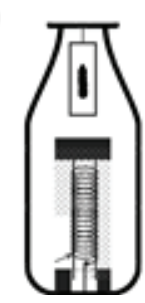

Figure 2

Modelo de detección de ozono: (a) Muestra testigo y (b) muestra con el ozonificador. 
En cada papel se impregnaron tres gotas de la mezcla de yoduro de potasio con agua. Luego se procede a introducir la muestra al interior del envase de la manera más rápida posible para evitar reacciones debidas al ozono del ambiente. El envase se limpia y desinfecta luego del análisis para poder repetir la experiencia.

\section{Resultados}

El proceso se repitió en cuatro ocasiones con diferentes tiempos, los cuales fueron: cinco, diez, quince y veinte minutos. En la Figura 3 se puede observar el resultado de la exposición con ozono para cada muestra.

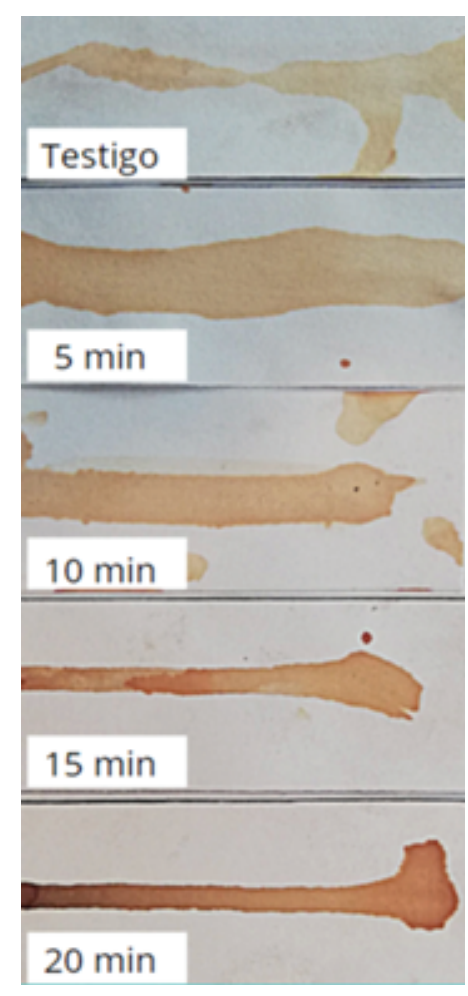

\section{Figure 3}

Muestras resultantes luego de la exposición con ozono.

Respuestas claras, directas y concisas a las preguntas u objetivos iniciales del.

Si realizamos una comparación cualitativa del cambio de coloración de la muestra con la Tabla 1 obtenemos resultados que nos pueden servir como una primera aproximación de la concentración de ozono en en el interior del envase.

En primera instancia podemos constatar que el color de la muestra a los veinte minutos es más oscura que en las anteriores experiencias. El código de color que le corresponde según la tabla 1 es la $\mathrm{D}$, entonces se puede inferir que en este intervalo de tiempo la concentración de ozono llega hasta unos supera los $210 \mu \mathrm{g} / \mathrm{m}^{\wedge} 3$. 


\section{Discusión}

La muestra testigo apenas logra la tonalidad de la muestra cuando pasan cinco minutos, pero dicho testigo tuvo una exposición más larga ( $20 \mathrm{~min}$ ). El cambio de tonalidad en la muestra testigo puede deberse a que el aire en la atmósfera tiene cierto porcentaje de ozono debido a una contaminación previa al experimento.

Se puede observar que cada intervalo de tiempo el tono de la muestra es cada vez más oscuro lo que implica una reacción en la cual se está produciendo Yodo con una velocidad comparable a cuando se tiene una barrera dieléctrica entre los electrodos (16.18). El aire está compuesto solo por un veinte por ciento de oxígeno por lo que es posible que el generador propuesto pueda producir más ozono, es decir, la masa final de producida está limitada por la cantidad de reactivo. Es por eso que al hacer una comparación con trabajos donde se trabajó con oxígeno puro se nota que existe mejor producción de ozono [14,19]. Se debe aclarar que se está realizando un análisis cualitativo de cada muestra observando el cambio de tono debido al yodo que se produce cuando el ozono reacciona con el yoduro de potasio reacción presentada en la introducción.

\section{Conclusiones}

Un núcleo generador de ozono con electrodos de estructura distintas fue propuesto como una alternativa a los generadores convencionales. Se encontró que tener un núcleo mixto no afecta el funcionamiento del mismo, demostrando que existe producción de ozono a pesar de las limitaciones presentadas. Al tener una combinación de dos tipos de electrodos en un generador nos permite aprovechar las características de cada uno por separado y así poder mejorar el funcionamiento del ozonificador.

Se puede realizar un futuro estudio de cómo afecta el consumo de energía de este tipo de núcleos mixtos. Además se pueden utilizar mejores métodos de detección de ozono para determinar la eficiencia y poder realizar una comparación con los ozonificadores actuales.

\section{Agradecimientos}

Es de mi menester expresar un cálido y fraterno agradecimiento bien merecido a $\mathrm{mi}$ abuelo Cesar Velasco, el cual fue un guía y apoyo importante en la realización de este trabajo, aportando con su basta experiencia y sabiendo resolver todo imprevisto.

\section{Conflicto de Intereses}

El único interés que me llevó a realizar el presente trabajo fue el de aportar con un grano de arena a la ciencia y enaltecer el nombre de mi institución. Además, me llamó mucho la atención esta oportunidad para poder tener una publicación que pueda aportar a mi perfil profesional. 


\section{References}

[1] Carey F, Velázquez A. Química orgánica. México: McGraw-Hill; 2006.

[2] Mordecai R. The history of ozone. The Schöbein period, 1839 - 1868. Bull Hist Chem. 2001;26:40-56.

[3] Peter C, Charles W; University of California. Electrolytic process for the production of ozone. United States patent US 4316782. 1982 Feb 23.

[4] Ferdes M, Zabava B, Dinca M, Paraschiv G. Effect of ozone treatment on three bacterial strains of drinking water. Jelgava. 2018;23:677-680.

[5] Hsieh P, Wen T. Evaluation of ozone removal by spent coffee grounds. Scientific Reports. 2020;10.

[6] González A. Generador de ozono por efecto corona para fines germicidas basado en un micro controlador con PWM. Paper presented at: Reunión Internacional de Otoño, ROC\&C; 2012 Nov 1115; Acapulco, México.

[7] Rozanov E. Preface: Ozone evolution in the past and future. Atmosphere. 2020;11:1-3.

[8] Gonçalves A. El ozono como agente antiséptico en la industria pesquera. Infopesca. 2007;31:32-37.

[9] Erazo L. Diseño y construcción de un ozonificador con capacidad de 300, para la purificación de ambientes. Santo Domingo de los Tsáchilas: UTE; 2012.

[10] López D, Hurtado J. Diseño e implementación de un prototipo generador de ozono para purificación de agua para el consumo humano. Quito: EPN; 2013.

[11] Vincent N, Sampson A, Akpama E. Analysis of Corona effect on transmission line. 2017;6:75-87.

[12] Conrad R; Conrad R. Methods of powering corona discharge in ozone generators. United States patent US 5130003. 1992 Jul 14.

[13] Sewell P, Luscombe R; NEWAIRE Inc. Ozone generator. United States US 5316741. 1994 May 31.

[14] Samaranayake W, Miyahara Y, Namihira T, Katsuki S, Hackam R, Akiyama H. Ozone generation in dry air using pulses discharges with and without a solid dielectric layer. IEEE Transaction on Dielectric and Electrical Insulations. 2001;8:687-697.

[15] Park S, Moon J, Lee S, Shin S. Effective ozone generation utilizing a meshed-plate electrode in a dielectric-barrier discharge type ozone generator. J of Electrostatic. 2005;64:275-282.

[16] Falcón Y, Meza J. Determinación de niveles de ozono en la atmósfera en la zona de Azcapotzalco. México DF: Universidad Autónoma Metropolitana; 1983.

[17] Shirk O. Las mediciones del ozono. MAPFRE. 2000;77:17-21.

[18] Kogelschatz U. Laser Surface Processing and Characterization. Boyd I, editor. Holanda: Elsevier Science Publisher; 1991. Silent-discharge driven excimer UV sources and their applications; p. 410-423.

[19] Pietsch G, Gibalov V. Dielectric barrier discharges and ozone synthesis. Pure \& Appl Chem. 1998; 70 : $1169-1174$. 